\title{
Optimization for Due-Date Assignment Single-Machine Scheduling under Group Technology
}

\author{
Li-Yan Wang $\left(\mathbb{D},{ }^{1}\right.$ Mengqi Liu $\left(\mathbb{D},{ }^{2}\right.$ Ji-Bo Wang $\left(\mathbb{D},{ }^{1}\right.$ Yuan-Yuan Lu $\mathbb{D}^{3},{ }^{3}$ and Wei-Wei Liu $\mathbb{D D}^{4}$ \\ ${ }^{1}$ School of Science, Shenyang Aerospace University, Shenyang 110136, China \\ ${ }^{2}$ Business School, Hunan University, Changsha 410082, Hunan, China \\ ${ }^{3}$ College of Mathematics, Jilin Normal University, Siping, Jilin, China \\ ${ }^{4}$ Department of Science, Shenyang Sport University, Shenyang 110102, China
}

Correspondence should be addressed to Mengqi Liu; liumengqi163@126.com

Received 9 November 2020; Revised 20 January 2021; Accepted 1 February 2021; Published 5 March 2021

Academic Editor: Lei Xie

Copyright $\odot 2021 \mathrm{Li}-Y$ an Wang et al. This is an open access article distributed under the Creative Commons Attribution License, which permits unrestricted use, distribution, and reproduction in any medium, provided the original work is properly cited.

\begin{abstract}
In this paper, the single-machine scheduling problem is studied by simultaneously considering due-date assignment and group technology (GT). The objective is to determine the optimal sequence of groups and jobs within groups and optimal due-date assignment to minimize the weighted sum of the absolute value in lateness and due-date assignment cost, where the weights are position dependent. For the common (CON) due-date assignment, slack (SLK) due-date assignment, and different (DIF) due-date assignment, an $O(n \log n)$ time algorithm is proposed, respectively, to solve the problem, where $n$ is the number of jobs.
\end{abstract}

\section{Introduction}

In the manufacturing industry, it is well known that firms can increase production efficiency by adopting the group technology (GT). The group technology is an approach to manufacturing that seeks to improve efficiency in highvolume production by exploiting the similarities of different products and activities in their production (Neufeld et al. [1] and Yang et al. [2]). Through decades of application, people have found many advantages of the group technology. For example, changeover between different jobs in the same group is simplified, reducing the costs or time involved; jobs in the same group spend less time waiting, which results in less work-in-process inventory; jobs in the same group tend to move through production in a direct route, reducing the manufacturing lead time (see the work of Yang and Yang [3], Lu et al. [4], Yin et al. [5], Wang and Liu [6], Wang and Wang [7], Qin et al. [8], Lu et al. [9], Zhang et al. [10], Liu et al. [11], and Wang and Liang [12]).

In recent years, the problem of the due-date assignment has been closely focused on by scholars (see the work of Yin et al. [13, 14], Wang et al. [15], and Shabtay [16]). Due to the increasing interest in the Just-In-Time (JIT) system, the issue of schedule allocation is becoming more and more important in practical applications. In the classical scheduling problem, the due date is usually a given constant, while in the actual application of life, the duration of the job is not a fixed constant, but a decision variable. In order to strengthen the global competition and improve the service level for customers, the jobs are required to be processed too early or too late in the JIT system, which leads to the trouble of scheduling problems involving advance and delay costs and the expiration date of the construction period. For the early completion of the job, it means that we have to bear a certain inventory cost, while for the delayed completion of the job, we have to bear the contract penalty, and the customer's goodwill is damaged (see the work of Li et al. [17], Liu et al. [18], Wang et al. [19]). A lot of literature deals with the problems such as the CON, SLK, and some other due-date assignment methods considering jobs. However, under the group technology, there are relatively few studies on the problem of the assignment of jobs. Li et al. [17] considered three due-date assignment methods under group technology. The objective function is to minimize the weighted sum of earliness, tardiness, due-date assignment, and completion time. For the CON, SLK, and different due-date assignments 
(DIFs), they proved that the problem can be solved in polynomial time, respectively. Ji et al. [20] studied the singlemachine slack due-date window assignment scheduling with group technology. The objective function is to minimize the total cost including the earliness, tardiness, due window starting time, and due window size. They proved that the problem can be solved in $O(n \log n)$ time.

Brucker [21] considered the CON due-date scheduling problem of minimizing the total cost comprising the total weighted absolute lateness value and common due-date $(\mathrm{CON})$ cost, where the weight is position dependent. He proved that the problem can be solved in a time $O(n \log n)$. Liu et al. [18] considered the SLK due-date assignment scheduling, and the goal is to minimize the total cost comprising the total weighted absolute lateness value and common flow allowance (SLK) cost, where the weight is a position-dependent weight. They proved that the problem can be solved in a time $O(n \log n)$. Wang et al. [19] studied the scheduling problems of single machine resource allocation in job-dependent learning effects. Under the linear and convex resource consumption functions, they proved that the CON and SLK due-date assignment problems can be solved in polynomial time, respectively. Sun et al. [22] considered single-machine scheduling problems on resource allocation, group technology, and learning effect. Under the linear and convex resource consumption functions, they proved that the SLK due-date assignment problem can be solved in polynomial time.

According to this study, we consider due-date assignment and scheduling problems with group technology. Under the assumption of group technology, the jobs are classified into groups by exploiting the similarities of different products and activities in their production. In this paper, we proceed to the study Brucker [21], Liu et al. [18], and Wang et al. [19], which is an extension of their work from considering the CON, SLK, and DIF due-date assignment scheduling problems with group technology and position-dependent weights. We organize the rest of the paper as follows: the problem is formulated in Section 2, several results of the optimal solution are introduced in Section 3, and the conclusions are summarized in Section 4.

\section{Problem Formulation}

In the study, the problem can be formally described as follows: there are $n$ independent non-preemption jobs grouped into $f$ groups, i.e., $\left\{G_{1}, G_{2}, \ldots, G_{f}\right\}$. A single machine and all the jobs are available at time zero, and the single machine can handle one job at a time and preemption is not allowed. Let the number of jobs in group $G_{i}$ be $n_{i}$, i.e., $\left\{J_{i, 1}, J_{i, 2}, \ldots, J_{i, n_{i}}\right\}$, where $J_{i, j}$ denotes the job $J_{j}$ of group $G_{i}$, $i=1,2, \ldots, f, j=1,2, \ldots, n_{i}$ and $n_{1}+n_{2}+\cdots+n_{f}=n$. Jobs in the same group are processed consecutively and do not need setup times. Let $p_{i j}$ denote the processing time of job $J_{i, j}$ and $s_{i}$ be the sequence-independent machine setup time incurred before the process of the first job of group $G_{i}$. Each job $J_{i, j}\left(i=1,2, \ldots, f ; j=1,2, \ldots, n_{i}\right)$ has a due date $d_{i, j}$, which is assignable according to one of the following three due-date assignment methods:
(1) The common $(\mathrm{CON})$ due-date assignment in which all jobs of group $G_{i}$ are assigned the same due date, i.e., $d_{i, j}=d_{i}^{\text {opt }}$ for $i=1,2, \ldots, f$ and $j=1,2, \ldots, n_{i}$

(2) The common flow allowance (slack, SLK) assignment in which the due-date $d_{i, j}$ for all jobs of group $G_{i}$ are assigned an equal flow allowance that is equal to its processing time plus the common flow allowance, i.e., $d_{i, j}=p_{i, j}+q_{i}^{\text {opt }}$ for $i=1,2, \ldots, f \quad$ and $j=1,2, \ldots, n_{i}$

(3) The different (DIF) due-date assignments in which the due date $d_{i, j}$ for all jobs of group $G_{i}$ are assigned a different due date with no restrictions $(i=1,2, \ldots, f$ and $j=1,2, \ldots, n_{i}$ )

Let $C_{i, j}$ be the completion time of job $J_{i, j}$. The goal is to determine the due date $d_{i, j}$ (i.e., the CON $d_{i}^{\text {opt }}$, the SLK $q_{i}^{\text {opt }}$, and the DIF $d_{i, j}$ ) and an optimal sequence $\pi^{*}$ such that the following objective functions are to be minimized:

$$
\begin{aligned}
& Z_{1}(\pi, D(\pi))=\sum_{i=1}^{f}\left(\sum_{j=1}^{n_{i}} \omega_{i j}\left|L_{\pi(i, j)}\right|+\omega_{i, 0} d_{i}^{\mathrm{opt}}\right), \\
& Z_{2}(\pi, D(\pi))=\sum_{i=1}^{f}\left(\sum_{j=1}^{n_{i}} \omega_{i, j}\left|L_{\pi(i, j)}\right|+\omega_{i, 0} q_{i}^{\mathrm{opt}}\right), \\
& Z_{3}(\pi, D(\pi))=\sum_{i=1}^{f} \sum_{j=1}^{n_{i}}\left(\omega_{i, j}\left|L_{\pi(i, j)}\right|+\omega_{i, 0} d_{\pi(i, j)}\right),
\end{aligned}
$$

where $\omega_{i, j}$ is the nonnegative weight of the $j$ th position in group $G_{i}$ (i.e., position-dependent weight, $i=1,2, \ldots, f ; j=1,2, \ldots$, $\left.n_{i}\right), \omega_{i, 0}$ denotes the unit cost of $d_{i}^{\text {opt }}\left(q_{i}^{\text {opt }}\right.$ and $\left.d_{\pi(i, j)}\right), i=$ $1,2, \ldots, f, \quad D(\pi)=\left(d_{1,1}(\pi), \ldots, d_{1, n_{1}}(\pi) ; \ldots ; d_{f, 1}(\pi), \ldots, d_{f, n_{f}}\right.$ $(\pi))$ is the due-date assignment vector under schedule $\pi$, and $L_{i, j}=C_{i, j}-d_{i, j}$ is the lateness of job $J_{i, j}$. Note that $\left|L_{\pi(i, j)}\right|=$ $E_{\pi(i, j)}+T_{\pi(i, j)}$, where $E_{\pi(i, j)}=\max \left\{0, d_{\pi(i, j)}-C_{\pi(i, j)}\right\}$ is the earliness of job $J_{i j}, T_{\pi(i, j)}=\max \left\{0, C_{\pi(i, j)}-d_{\pi(i, j)}\right\}$ is the tardiness of job $J_{i j}$, and $\pi(i, j)$ represents the job that is in the $j$ th position of group $G_{i}$ in $\pi$ for $1 \leq k \leq n_{i}$. By using the three-field notation (see Graham et al. [23]), the problems can be denoted by

$$
\begin{aligned}
& 1|\mathrm{GT}, \mathrm{CON}| \sum_{i=1}^{f}\left(\sum_{j=1}^{n_{i}} \omega_{i j}\left|L_{\pi(i, j)}\right|+\omega_{i, 0} d_{i}^{\mathrm{opt}}\right), \\
& 1|\mathrm{GT}, \mathrm{SLK}| \sum_{i=1}^{f}\left(\sum_{j=1}^{n_{i}} \omega_{i, j}\left|L_{\pi(i, j)}\right|+\omega_{i, 0} q_{i}^{\mathrm{opt}}\right), \\
& 1|\mathrm{GT}, \mathrm{DIF}| \sum_{i=1}^{f} \sum_{j=1}^{n_{i}}\left(\omega_{i, j}\left|L_{\pi(i, j)}\right|+\omega_{i, 0} d_{\pi(i, j)}\right),
\end{aligned}
$$

respectively, where GT denotes the group technology.

\section{Main Results}

Lemma 1. For each of the three due-date assignment methods, there exists an optimal schedule with zero machine idle times. 
Proof. It is omitted due to simplicity.

Therefore, it is convenient to introduce a dummy job $J_{i, 0}$ of group $G_{i}$ for $i=1,2, \ldots, f$ with processing time $p_{i, 0}=0$ and weight $\omega_{i, 0}$ which is always scheduled at time 0 , i.e., $\pi(i, 0)=0$. Consequently, for any given job schedule $\pi$, the completion times can be calculated by the following equation:

$$
C_{\pi(i, j)}=S_{i}(\pi)+s_{i}+\sum_{k=1}^{j} p_{\pi(i, k)},
$$

where $S_{i}(\pi)$ denotes the starting time of group $G_{i}$ in schedule $\pi$ for $i=1,2, \ldots, f$.

Lemma 2. If the sequence of groups is fixed, for the CON and SLK due-date assignment methods, there exists an optimal sequence with the property that $d_{i}^{\text {opt }}\left(q_{i}^{\text {opt }}\right)$ coincides with the job completion times of group $G_{i}$ for $i=1,2, \ldots, f$.

Proof. Let the sequence of groups be fixed. Consider an optimal sequence $\pi$ in $G_{i}$.

(a) First, we prove that the common due-date $d_{i}^{\text {opt }}$ of group $G_{i}$ coincides with the completion time of a job of group $G_{i}$. Assume that $C_{\pi\left(i, k_{i}\right)}<d_{i}^{\text {opt }}<C_{\pi\left(i, k_{i}+1\right)}$; therefore,

$$
\begin{aligned}
Z_{1 i}\left(\pi, D_{i}(\pi)\right) & =\sum_{j=1}^{n_{i}} \omega_{i, j}\left|L_{\pi(i, j)}\right|+\omega_{i, 0} d_{i}^{\mathrm{opt}} \\
& =\sum_{j=1}^{n_{i}} \omega_{i, j}\left|C_{\pi(i, j)}-d_{i}^{\mathrm{opt}}\right|+\omega_{i, 0} d_{i}^{\mathrm{opt}}
\end{aligned}
$$

$$
\begin{aligned}
= & \sum_{j=1}^{k_{i}} \omega_{i, j}\left(d_{i}^{\mathrm{opt}}-C_{\pi(i, j)}\right) \\
& +\sum_{j=k_{i}+1}^{n_{i}} \omega_{i, j}\left(C_{\pi(i, j)}-d_{i}^{\mathrm{opt}}\right)+\omega_{i, 0} d_{i}^{\mathrm{opt}} .
\end{aligned}
$$

If $d_{i}^{\text {opt }}=C_{\pi\left(i, k_{i}\right)}$, we have

$$
\begin{aligned}
Z_{1 i}^{1}\left(\pi, D_{i}(\pi)\right)= & \sum_{j=1}^{k_{i}} \omega_{i, j}\left(C_{\pi\left(i, k_{i}\right)}-C_{\pi(i, j)}\right) \\
& +\sum_{j=k_{i}+1}^{n_{i}} \omega_{i, j}\left(C_{\pi(i, j)}-C_{\pi\left(i, k_{i}\right)}\right) \\
& +\omega_{i, 0} C_{\pi\left(i, k_{i}\right)} .
\end{aligned}
$$

If $d_{i}^{\text {opt }}=C_{\pi\left(i, k_{i}+1\right)}$, we have

$$
\begin{aligned}
Z_{1 i}^{2}\left(\pi, D_{i}(\pi)\right)= & \sum_{j=1}^{k_{i}} \omega_{i, j}\left(C_{\pi\left(i, k_{i}+1\right)}-C_{\pi(i, j)}\right) \\
& +\sum_{j=k_{i}+1}^{n_{i}} \omega_{i, j}\left(C_{\pi(i, j)}-C_{\pi\left(i, k_{i}+1\right)}\right) \\
& +\omega_{i, 0} C_{\pi\left(i, k_{i}+1\right)} .
\end{aligned}
$$

Let $\quad x=d_{i}^{\mathrm{opt}}-C_{\pi\left(i, k_{i}\right)}>0, \quad y=C_{\pi\left(i, k_{i}+1\right)}-d_{i}^{\mathrm{opt}}>0$, then we can obtain that

$$
\begin{aligned}
& Z_{1 i}\left(\pi, D_{i}(\pi)\right)-Z_{1 i}^{1}\left(\pi, D_{i}(\pi)\right), \\
& =\sum_{j=1}^{k_{i}} \omega_{i, j}\left(d_{i}^{\mathrm{opt}}-C_{\pi\left(i, k_{i}\right)}\right)+\sum_{j=k_{i}+1}^{n_{i}} \omega_{i, j}\left(C_{\pi\left(i, k_{i}\right)}-d_{i}^{\mathrm{opt}}\right)+\omega_{i, 0}\left(d_{i}^{\mathrm{opt}}-C_{\pi\left(i, k_{i}\right)}\right), \\
& =\sum_{j=0}^{k_{i}} \omega_{i, j}\left(d_{i}^{\mathrm{opt}}-C_{\pi\left(i, k_{i}\right)}\right)+\sum_{j=k_{i}+1}^{n_{i}} \omega_{i, j}\left(C_{\pi\left(i, k_{i}\right)}-d_{i}^{\mathrm{opt}}\right), \\
& =x\left(\sum_{j=0}^{k_{i}} \omega_{i, j}-\sum_{j=k_{i}+1}^{n_{i}} \omega_{i, j}\right), \\
& Z_{1 i}\left(\pi, D_{i}(\pi)\right)-Z_{1 i}^{2}\left(\pi, D_{i}(\pi)\right), \\
& =\sum_{j=1}^{k_{i}} \omega_{i, j}\left(d_{i}^{\mathrm{opt}}-C_{\pi\left(i, k_{i}+1\right)}\right)+\sum_{j=k_{i}+1}^{n_{i}} \omega_{i, j}\left(C_{\pi\left(i, k_{i}+1\right)}-d_{i}^{\mathrm{opt}}\right)+\omega_{i, 0}\left(d_{i}^{\mathrm{opt}}-C_{\pi\left(i, k_{i}+1\right)}\right), \\
& =\sum_{j=0}^{k_{i}} \omega_{i, j}\left(d_{i}^{\mathrm{opt}}-C_{\pi\left(i, k_{i}+1\right)}\right)+\sum_{j=k_{i}+1}^{n_{i}} \omega_{i, j}\left(C_{\pi\left(i, k_{i}+1\right)}-d_{i}^{\mathrm{opt}}\right), \\
& =-y\left(\sum_{j=0}^{k_{i}} \omega_{i, j}-\sum_{j=k_{i}+1}^{n_{i}} \omega_{i, j}\right) .
\end{aligned}
$$


4

Complexity

Thus, $Z_{1 i}^{1}\left(\pi, D_{i}(\pi)\right) \leq Z_{1 i}\left(\pi, D_{i}(\pi)\right)$ if $\sum_{j=0}^{k_{i}} \omega_{i, j} \geq$ $\sum_{j=k_{i}+1}^{n_{i}} \omega_{i, j} \quad$ and $\quad Z_{1 i}^{2}\left(\pi, D_{i}(\pi)\right) \leq Z_{1 i}\left(\pi, D_{i}(\pi)\right)$ otherwise. (b) Assume that $C_{\pi\left(i, l_{i}\right)}<q_{i}^{\text {opt }}<C_{\pi\left(i, l_{i}+1\right)}$; therefore,

$$
\begin{aligned}
Z_{2 i}(\pi) & =\sum_{j=1}^{n_{i}} \omega_{i, j}\left|L_{\pi(i, j)}\right|+\omega_{i, 0} q_{i}^{\mathrm{opt}}, \\
& =\sum_{j=1}^{n_{i}} \omega_{i, j}\left|C_{\pi(i, j)}-p_{\pi(i, j)}-q_{i}^{\mathrm{opt}}\right|+\omega_{i, 0} q_{i}^{\mathrm{opt}}, \\
& =\sum_{j=1}^{n_{i}} \omega_{i, j}\left|C_{\pi(i, j-1)}-q_{i}^{\mathrm{opt}}\right|+\omega_{i, 0} q_{i}^{\mathrm{opt}}, \\
& =\sum_{j=1}^{l_{i}+1} \omega_{i, j}\left(q_{i}^{\mathrm{opt}}-C_{\pi(i, j-1)}\right)+\sum_{j=l_{i}+2}^{n_{i}} \omega_{i, j}\left(C_{\pi(i, j-1)}-q_{i}^{\mathrm{opt}}\right)+\omega_{i, 0} q_{i}^{\mathrm{opt}},
\end{aligned}
$$

where $C_{\pi(i, 0)}=S_{i}(\pi)+s_{i}$.

If $q_{i}^{\text {opt }} \stackrel{=}{=} C_{\pi\left(i, l_{i}\right)}$, we have

$$
\begin{aligned}
Z_{2 i}^{\prime}(\pi)= & \sum_{j=1}^{l_{i}+1} \omega_{i, j}\left(C_{\pi\left(i, l_{i}\right)}-C_{\pi(i, j-1)}\right) \\
& +\sum_{j=l_{i}+2}^{n_{i}} \omega_{i, j}\left(C_{\pi(i, j-1)}-C_{\pi\left(i, l_{i}\right)}\right)+\omega_{i, 0} C_{\pi\left(i, l_{i}\right)}
\end{aligned}
$$

$$
\begin{aligned}
Z_{2 i}^{\prime \prime}(\pi)= & \sum_{j=1}^{l_{i}+1} \omega_{i, j}\left(C_{\pi\left(i, l_{i}+1\right)}-C_{\pi(i, j-1)}\right) \\
& +\sum_{j=l_{i}+2}^{n_{i}} \omega_{i, j}\left(C_{\pi(i, j-1)}-C_{\pi\left(i, l_{i}+1\right)}\right)+\omega_{i, 0} C_{\pi\left(i, l_{i}+1\right)}
\end{aligned}
$$

Let $x^{\prime}=q_{i}^{\mathrm{opt}}-C_{\pi\left(i, l_{i}\right)}>0$ and $y^{\prime}=C_{\pi\left(i, l_{i}+1\right)}-q_{i}^{\mathrm{opt}}>0$; then, we have

If $q_{i}^{\text {opt }}=C_{\pi\left(i, l_{i}+1\right)}$, we have

$$
\begin{aligned}
& Z_{2 i}\left(\pi, D_{i}(\pi)\right)-Z_{2 i}^{\prime}\left(\pi, D_{i}(\pi)\right), \\
&= \sum_{j=1}^{l_{i}+1} \omega_{i, j}\left(q_{i}^{\mathrm{opt}}-C_{\pi\left(i, l_{i}\right)}\right)+\sum_{j=l_{i}+2}^{n_{i}} \omega_{i, j}\left(C_{\pi\left(i, l_{i}\right)}-q_{i}^{\mathrm{opt}}\right)+\omega_{i, 0}\left(q_{i}^{\mathrm{opt}}-C_{\pi\left(i, l_{i}\right)}\right), \\
&= \sum_{j=0}^{l_{i}+1} \omega_{i, j}\left(q_{i}^{\mathrm{opt}}-C_{\pi\left(i, l_{i}\right)}\right)+\sum_{j=l_{i}+2}^{n_{i}} \omega_{i, j}\left(C_{\pi\left(i, l_{i}\right)}-q_{i}^{\mathrm{opt}}\right), \\
&= x^{\prime}\left(\sum_{j=0}^{l_{i}+1} \omega_{i, j}-\sum_{j=l_{i}+2}^{n_{i}} \omega_{i, j}\right), \\
& Z_{2 i}\left(\pi, D_{i}(\pi)\right)-Z_{2 i}^{\prime \prime}\left(\pi, D_{i}(\pi)\right), \\
&=\sum_{j=1}^{l_{i}+1} \omega_{i, j}\left(q_{i}^{\mathrm{opt}}-C_{\pi\left(i, l_{i}+1\right)}\right)+\sum_{j=l_{i}+2}^{n_{i}} \omega_{i, j}\left(C_{\pi\left(i, l_{i}+1\right)}-q_{i}^{\mathrm{opt}}\right)+\omega_{i, 0}\left(q_{i}^{\mathrm{opt}}-C_{\pi\left(i, l_{i}+1\right)}\right), \\
&=\sum_{j=0}^{l_{i}+1} \omega_{i, j}\left(q_{i}^{\mathrm{opt}}-C_{\pi\left(i, l_{i}+1\right)}\right)+\sum_{j=l_{i}+2}^{n_{i}} \omega_{i, j}\left(C_{\pi\left(i, l_{i}+1\right)}-q_{i}^{\mathrm{opt}}\right), \\
&=-y^{\prime}\left(\sum_{j=0}^{l_{i}+1} \omega_{i, j}-\sum_{j=l_{i}+2}^{n_{i}} \omega_{i, j}\right) .
\end{aligned}
$$


Thus, $Z_{2 i}^{\prime}\left(\pi, D_{i}(\pi)\right) \leq Z_{2 i}\left(\pi, D_{i}(\pi)\right)$ if $\sum_{j=0}^{l_{i}+1} \omega_{i, j} \geq \sum_{j=l_{i}+2}^{n_{i}}$ $\omega_{i, j}$ and $Z_{2 i}^{\prime \prime}\left(\pi, D_{i}(\pi)\right) \leq Z_{2 i}\left(\pi, D_{i}(\pi)\right)$ otherwise.

From (a) and (b), this implies that $d_{i}^{\text {opt }}\left(q_{i}^{\text {opt }}\right)$ is equal to the job completion times of group $G_{i}$ for $i=1,2, \ldots, f$.

Lemma 3. For the CON due-date assignment, if the sequence of groups is fixed, for a given schedule $\pi$, there exists an optimal schedule of group $G_{i}$ in which $d_{i}^{\text {opt }}=C_{\pi\left(i, k_{i}\right)}$, where $k_{i}$ is a median for the sequence $\omega_{i, 0}, \omega_{i, 1}, \ldots, \omega_{i, n_{i}}$ and $\sum_{j=0}^{k_{i}-1} \omega_{i, j} \leq \sum_{j=k_{i}}^{n_{i}} \omega_{i, j}$ and $\sum_{j=0}^{k_{i}} \omega_{i, j} \geq \sum_{j=k_{i}+1}^{n_{i}} \omega_{i, j}$.

Proof. By using the technique of small perturbations, from Lemma 2, we assume that $d_{i}^{\mathrm{opt}}=C_{\pi\left(i, k_{i}\right)}$. Applying (7) and (8) to the cases of moving the common due-date $x$ units of time to the left (right), we have $\sum_{j=k_{i}}^{n_{i}} \omega_{i, j}-\sum_{j=0}^{k_{i}-1} \omega_{i, j} \geq 0$ and $\sum_{j=0}^{k_{i}} \omega_{i, j}-\sum_{j=k_{i}+1}^{n_{i}} \omega_{i, j} \geq 0$.

Lemma 4. For the SLK due-date assignment, if the sequence of groups is fixed, for a given schedule $\pi$, there exists an optimal schedule of group $G_{i}$ in which $q_{i}^{\text {opt }}=C_{\pi\left(i, l_{i}\right)}$, where $l_{i}$ is a median for the sequence $\omega_{i, 0}, \omega_{i, 1}, \ldots, \omega_{i, n_{i}}$ and $\sum_{j=0}^{l_{i}} \omega_{i, j} \leq \sum_{j=l_{i}+1}^{n_{i}} \omega_{i, j}$ and $\sum_{j=0}^{l_{i}+1} \omega_{i, j} \geq \sum_{j=l_{i}+2}^{n_{i}} \omega_{i, j}$.

Proof. It is similar to the proof of Lemma 3.
Lemma 5. For the DIF due-date assignment method, there exists an optimal sequence such that $d_{\pi(i, j)} \leq C_{\pi(i, j)}$.

Proof. We consider the case that contradicts this optimal property. Consider an optimal sequence $\pi$ in $G_{i}$, if $C_{\pi(i, j)}<d_{\pi(i, j)}$, and then, the total cost for job $J_{\pi(i, j)}$ is

$$
Z_{\pi(i, j)}=\omega_{i, j}\left(d_{\pi(i, j)}-C_{\pi(i, j)}\right)+\omega_{i, 0} d_{\pi(i, j)} .
$$
we have

We shift $d_{\pi(i, j)}$ to the left such that $d_{\pi(i, j)}=C_{\pi(i, j)}$, and

$$
\widetilde{Z}_{\pi(i, j)}=\omega_{i, 0} d_{\pi(i, j)}<Z_{\pi(i, j)} .
$$

Hence, the case $C_{\pi(i, j)}<d_{\pi(i, j)}$ is not an optimal due-date assignment. Lemma 5 is proved.

Lemma 6. For the DIF due-date assignment method, let $\pi$ be a given sequence, and the optimal due-date $d_{\pi(i, j)}$ for job $J_{\pi(i, j)}$ can be obtained as follows:

$$
d_{\pi(i, j)}= \begin{cases}0, & \omega_{i, j} \leq \omega_{i, 0} \\ C_{\pi(i, j)}, & \omega_{i, j}>\omega_{i, 0} .\end{cases}
$$

Proof. From Lemma 5, for job $J_{\pi(i, j)}$, we have

$$
Z_{\pi(i, j)}=\omega_{i, j}\left(C_{\pi(i, j)}-d_{\pi(i, j)}\right)+\omega_{i, 0} d_{\pi(i, j)}=\omega_{i, j} C_{\pi(i, j)}+\left(\omega_{i, 0}-\omega_{i, j}\right) d_{\pi(i, j)} .
$$

Obviously, if $\omega_{i, j} \leq \omega_{i, 0}, d_{\pi(i, j)}$ should be 0 , if $\omega_{i, j}>\omega_{i, 0} d_{\pi(i, j)}$ should be $C_{\pi(i, j)}$. Hence, the total cost for job $J_{\pi(i, j)}$ is

$$
Z_{\pi(i, j)}=\eta_{i, j} C_{\pi(i, j)},
$$

where $\eta_{i, j}=\min \left\{\omega_{i, j}, \omega_{i, 0}\right\}$.
For the CON due-date assignment, obviously, the value of $k_{i}(i=1,2, \ldots, f)$ given in Lemma 3 is independent of the job processing times and the job sequence. Therefore, it is optimal for any job sequence within each group. For a given schedule $\pi, d_{i}^{\mathrm{opt}}=C_{\pi\left(i, k_{i}\right)}$, and the total cost of all the jobs within $G_{i}$ for $i=1, \ldots, m$ is given by

$$
\begin{aligned}
Z_{1 i}(\pi) & =\sum_{j=1}^{n_{i}} \omega_{i, j}\left|L_{\pi(i, j)}\right|+\omega_{i, 0} d_{i}^{\mathrm{opt}}, \\
& =\sum_{j=1}^{n_{i}} \omega_{i, j}\left|C_{\pi(i, j)}-d_{i}^{\mathrm{opt}}\right|+\omega_{i, 0} d_{i}^{\mathrm{opt}}, \\
& =\omega_{i, 0} C_{\pi\left(i, k_{i}\right)}+\sum_{j=1}^{k_{i}} \omega_{i, j}\left(C_{\pi\left(i, k_{i}\right)}-C_{\pi(i, j)}\right)+\sum_{j=k_{i}+1}^{n_{i}} \omega_{i, j}\left(C_{\pi(i, j)}-C_{\pi\left(i, k_{i}\right)}\right), \\
& =\omega_{i, 0}\left(S_{i}+s_{i}\right)+\sum_{j=0}^{k_{i}} \omega_{i, j}\left(\sum_{h=j+1}^{k_{i}} p_{\pi(i, h)}\right)+\sum_{j=k_{i}+1}^{n_{i}} \omega_{i, j}\left(\sum_{h=k_{i}+1}^{j} p_{\pi(i, h)}\right), \\
& =\sum_{h=1}^{k_{i}} p_{\pi(i, h)}\left(\sum_{j=0}^{h-1} \omega_{i, j}\right)+\sum_{h=k_{i}+1}^{n_{i}} p_{\pi(i, h)}\left(\sum_{j=h}^{n_{i}} \omega_{i, j}\right)+\omega_{i, 0}\left(S_{i}+s_{i}\right), \\
& =\sum_{j=1}^{n_{i}} W_{i, j} p_{\pi(i, j)}+\omega_{i, 0}\left(S_{i}+s_{i}\right),
\end{aligned}
$$


where

$$
W_{i, j}= \begin{cases}\sum_{h=0}^{j-1} \omega_{i, h}, & j=1,2, \ldots, k_{i}, \\ \sum_{h=j}^{n_{i}} \omega_{i, h}, & j=k_{i}+1, \ldots, n_{i} .\end{cases}
$$

For the SLK due-date assignment, obviously, the value of $l_{i}(i=1,2, \ldots, f)$ given in Lemma 4 is independent of the job processing times and the job sequence. Therefore, it is optimal for any job sequence within each group. For a given schedule $\pi, q_{i}^{\text {opt }}=C_{\pi\left(i, l_{i}\right)}$, and the total cost of all the jobs within $G_{i}$ for $i=1,2, \ldots, f$ is given by

$$
\begin{aligned}
Z_{2 i}(\pi) & =\sum_{j=1}^{n_{i}} \omega_{i, j}\left|L_{\pi(i, j)}\right|+\omega_{i, 0} q_{i}^{\mathrm{opt}}, \\
& =\sum_{j=1}^{n_{i}} \omega_{i, j}\left|C_{\pi(i, j)}-p_{\pi(i, j)}-q_{i}^{\mathrm{opt}}\right|+\omega_{i, 0} q_{i}^{\mathrm{opt}}, \\
& =\sum_{j=1}^{n_{i}} \omega_{i, j}\left|C_{\pi(i, j-1)}-q_{i}^{\mathrm{opt}}\right|+\omega_{i, 0} q_{i}^{\mathrm{opt}}, \\
& =\sum_{j=1}^{n_{i}} \omega_{i, j}\left|C_{\pi(i, j-1)}-C_{\pi\left(i, l_{i}\right)}\right|+\omega_{i, 0} C_{\pi\left(i, l_{i}\right)} \\
& =\sum_{j=1}^{l_{i}+1} \omega_{i, j}\left(C_{\pi\left(i, l_{i}\right)}-C_{\pi(i, j-1)}\right)+\sum_{j=l_{i}+2}^{n_{i}} \omega_{i, j}\left(C_{\pi(i, j-1)}-C_{\pi\left(i, l_{i}\right)}\right)+\omega_{i, 0} C_{\pi\left(i, l_{i}\right)}, \\
& =\sum_{j=0}^{l_{i}+1} \omega_{i, j}\left(\sum_{h=j}^{l_{i}} p_{\pi(i, h)}\right)+\sum_{j=l_{i}+2}^{n_{i}} \omega_{i, j}\left(\sum_{h=l_{i}+1}^{j-1} p_{\pi(i, h)}\right)+\omega_{i, 0}\left(S_{i}+s_{i}\right), \\
& =\sum_{j=1}^{l_{i}} p_{\pi(i, j)}\left(\sum_{h=0}^{j} \omega_{i, h}\right)+\sum_{j=l_{i}+1}^{n_{i}-1} p_{\pi(i, j)}\left(\sum_{h=j+1}^{n_{i}} \omega_{i, h}\right)+\omega_{i, 0}\left(S_{i}+s_{i}\right), \\
= & \sum_{j=1}^{n_{i}} V_{i, j} p_{\pi(i, j)}+\omega_{i, 0}\left(S_{i}+s_{i}\right),
\end{aligned}
$$

where

$$
V_{i, j}= \begin{cases}\sum_{h=0}^{j-1} \omega_{i, h}, & j=1,2, \ldots, l_{i}, \\ \sum_{h=j+1}^{n_{i}} \omega_{i, h}, & j=l_{i}+1, \ldots, n_{i}-1, \\ 0, & j=n_{i} .\end{cases}
$$

For the DIF due-date assignment, from Lemma 6, the total cost of all the jobs within $G_{i}$ for $i=1,2, \ldots, f$ is given by

$$
\begin{aligned}
Z_{3 i}(\pi) & =\sum_{j=1}^{n_{i}}\left(\omega_{i, j}\left|L_{\pi(i, j)}\right|+\omega_{i, 0} d_{\pi(i, j)}\right), \\
& =\sum_{j=1}^{n_{i}} \eta_{i, j} C_{\pi(i, j)}, \\
& =\sum_{j=1}^{n_{i}} \eta_{i, j}\left(S_{i}+s_{i}+\sum_{h=1}^{j} p_{\pi(i, h)}\right), \\
& =\sum_{j=1}^{n_{i}} U_{i, j} p_{\pi(i, j)}+\left(S_{i}+s_{i}\right) \sum_{j=1}^{n_{i}} \omega_{i, j},
\end{aligned}
$$

where

$$
U_{i, j}=\sum_{h=j}^{n_{i}} \eta_{i, h}, \quad h=1,2, \ldots, n_{i} .
$$

Obviously, from (18), (20), and (22), the term $\sum_{j=1}^{n_{i}} W_{i, j} p_{\pi(i, j)}\left(\sum_{j=1}^{n_{i}} V_{i, j} p_{\pi(i, j)}\right.$ and $\left.\sum_{j=1}^{n_{i}} U_{i, j} p_{\pi(i, j)}\right)$ is only concerned with the job processing sequence within group $G_{i}$ and can be minimized by the HLP rule (see the work of Hardy et al. [24]), i.e., the optimal job sequence within group $G_{i}(i=1,2, \ldots, f)$ can be obtained by arranging the elements of the $W_{i, j}\left(V_{i, j}\right.$ and $\left.U_{i, j}\right)$ and $p_{i, j}$ vectors in opposite orders. The term $\omega_{i, 0}\left(S_{i}+s_{i}\right)\left(\left(S_{i}+s_{i}\right) \sum_{j=1}^{n_{i}} \omega_{i, j}\right)$ is only dependent on the starting time of the group $G_{i}$, and the optimal sequence of the groups $\left\{G_{1}, G_{2}, \ldots, G_{f}\right\}$ can be obtained by the following lemma.

Lemma 7. For the problems $1|\mathrm{GT}, \mathrm{CON}| \sum_{i=1}^{f}\left(\sum_{j=1}^{n_{i}} \omega_{i j}\left|L_{\pi(i, j)}\right|\right.$ $\left.+\omega_{i, 0} d_{i}^{\text {opt }}\right)$ and $1|\mathrm{GT}, \mathrm{SLK}| \sum_{i=1}^{f}\left(\sum_{j=1}^{n_{i}} \omega_{i, j}\left|L_{\pi(i, j)}\right|+\omega_{i, 0} q_{i}^{\mathrm{opt}}\right)$, the optimal group sequence can be obtained by arranging the groups in nondecreasing order of $\theta_{i}=\left(s_{i}+\sum_{j=1}^{n_{i}} p_{i, j}\right) \omega_{i, 0}, i=1,2, \ldots, f$, respectively. For the problem $1|\mathrm{GT}, \mathrm{DIF}| \sum_{i=1}^{f} \sum_{j=1}^{n_{i}}\left(\omega_{i, j}\left|L_{\pi(i, j)}\right|\right.$ $\left.+\omega_{i, 0} d_{\pi(i, j)}\right)$, the optimal group sequence can be obtained by 
arranging the groups in nondecreasing order of $\theta_{i}=\left(s_{i}+\right.$ $\left.\sum_{j=1}^{n_{i}} p_{i, j}\right) \sum_{j=1}^{n_{i}} \omega_{i, j}, i=1,2, \ldots, f$.

Proof. For the problem $1|\mathrm{GT}, \mathrm{CON}| \sum_{i=1}^{f}\left(\sum_{j=1}^{n_{i}} \omega_{i j}\right.$ $\left.\left|L_{\pi(i, j)}\right|+\omega_{i, 0} d_{i}^{\text {opt }}\right)$, let $\pi$ and $\pi^{\prime}$ be two sequences where the difference between $\pi$ and $\pi^{\prime}$ is a pairwise interchange of two adjacent groups $G_{k}$ and $G_{l}$, that is, $\pi=\left[A, G_{k}, G_{l}, B\right]$ and $\pi^{\prime}=\left[A, G_{l}, G_{k}, B\right]$, where $A$ and $B$ are partial sequences. To show $\pi$ dominates $\pi^{\prime}$, it suffices to show that $Z_{1}(\pi, D(\pi)) \leq Z_{1}\left(\pi^{\prime}, D\left(\pi^{\prime}\right)\right)$. We assume that $S$ denotes the completion time of the last job in $A$, and we have

$$
\begin{gathered}
S_{l}(\pi)=S+s_{k}+\sum_{j=1}^{n_{k}} p_{k, j}, \\
S_{k}\left(\pi^{\prime}\right)=S+s_{l}+\sum_{j=1}^{n_{l}} p_{l, j} .
\end{gathered}
$$

Suppose that

$$
\frac{s_{k}+\sum_{j=1}^{n_{k}} p_{k, j}}{\omega_{k, 0}} \leq \frac{s_{l}+\sum_{j=1}^{n_{l}} p_{l, j}}{\omega_{l, 0}},
$$

and from (18), we have

$$
\begin{aligned}
Z_{1} & (\pi, D(\pi))-Z_{1}\left(\pi^{\prime}, D\left(\pi^{\prime}\right)\right), \\
& =\omega_{k, 0}\left(S+s_{k}\right)+\omega_{l, 0}\left(S+s_{k}+\sum_{j=1}^{n_{k}} p_{k j}+s_{l}\right) \\
& -\omega_{l, 0}\left(S+s_{l}\right)-\omega_{k, 0}\left(S+s_{l}+\sum_{j=1}^{n_{l}} p_{l j}+s_{k}\right), \\
& =\omega_{l, 0}\left(s_{k}+\sum_{j=1}^{n_{k}} p_{k, j}\right)-\omega_{k, 0}\left(s_{l}+\sum_{j=1}^{n_{l}} p_{l, j}\right) \\
& \leq 0 .
\end{aligned}
$$

Therefore, $Z_{1}(\pi, D(\pi)) \leq Z_{1}\left(\pi^{\prime}, D\left(\pi^{\prime}\right)\right)$. This completes the proof of the problem $1|\mathrm{GT}, \mathrm{CON}| \sum_{i=1}^{f}\left(\sum_{j=1}^{n_{i}} \omega_{i j}\right.$ $\left.\left|L_{\pi(i, j)}\right|+\omega_{i, 0} d_{i}^{\mathrm{opt}}\right)$.

For the problem $1|\mathrm{GT}, \mathrm{SLK}| \sum_{i=1}^{f}\left(\sum_{j=1}^{n_{i}} \omega_{i j}\left|L_{\pi(i, j)}\right|\right.$ $+\omega_{i, 0} q_{i}^{\text {opt }}$, the proof can be obtained similarly.

For the problem $1|\mathrm{GT}, \mathrm{DIF}| \sum_{i=1}^{f}\left(\sum_{j=1}^{n_{i}} \omega_{i j}\left|L_{\pi(i, j)}\right|\right.$ $\left.+\omega_{i, 0} d_{i}^{\mathrm{opt}}\right)$, stemming from the proof of the problem $1|\mathrm{GT}, \mathrm{CON}| \sum_{i=1}^{f}\left(\sum_{j=1}^{n_{i}} \omega_{i j}\left|L_{\pi(i, j))}\right|+\omega_{i, 0} d_{i}^{\mathrm{opt}}\right)$, we suppose that

$$
\frac{s_{k}+\sum_{j=1}^{n_{i}} p_{k, j}}{\sum_{j=1}^{n_{k}} \omega_{k, j}} \leq \frac{s_{l}+\sum_{j=1}^{n_{i}} p_{l, j}}{\sum_{j=1}^{n_{l}} \omega_{l, j}},
$$

and from (22), we have

$$
\begin{aligned}
Z_{3} & (\pi, D(\pi))-Z_{3}\left(\pi^{\prime}, D\left(\pi^{\prime}\right)\right), \\
& =\left(S+s_{k}\right) \sum_{j=1}^{n_{k}} \omega_{k, j}+\left(S+s_{k}+\sum_{j=1}^{n_{k}} p_{k j}+s_{l}\right) \sum_{j=1}^{n_{l}} \omega_{l, j}-\left(S+s_{l}\right) \sum_{j=1}^{n_{l}} \omega_{l, j}-\left(S+s_{l}+\sum_{j=1}^{n_{l}} p_{l j}+s_{k}\right) \sum_{j=1}^{n_{k}} \omega_{k, j}, \\
& =\sum_{j=1}^{n_{l}} \omega_{l, j}\left(s_{k}+\sum_{j=1}^{n_{k}} p_{k, j}\right)-\sum_{j=1}^{n_{k}} \omega_{k, j}\left(s_{l}+\sum_{j=1}^{n_{l}} p_{l, j}\right) \\
& \leq 0 .
\end{aligned}
$$

Therefore, $Z_{1}(\pi, D(\pi)) \leq Z_{1}\left(\pi^{\prime}, D\left(\pi^{\prime}\right)\right)$. This completes the proof of the problem $1|\mathrm{GT}, \mathrm{CON}| \sum_{i=1}^{f}\left(\sum_{j=1}^{n_{i}} \omega_{i j}\left|L_{\pi(i, j)}\right|\right.$ $\left.+\omega_{i, 0} d_{i}^{\mathrm{opt}}\right)$.

Based on the abovementioned analysis, we can present the following algorithm to solve the problems $1|\mathrm{GT}, \mathrm{CON}|$ $\sum_{i=1}^{f}\left(\sum_{j=1}^{n_{i}} \omega_{i j}\left|L_{\pi(i, j)}\right|+\omega_{i, 0} d_{i}^{\mathrm{opt}}\right), \quad 1|\mathrm{GT}, \mathrm{SLK}| \sum_{i=1}^{f}\left(\sum_{j=1}^{n_{i}} \omega_{i, j}\right.$ $\left.\left|L_{\pi(i, j)}\right|+\omega_{i, 0} q_{i}^{\mathrm{opt}}\right)$, and $1|\mathrm{GT}, \mathrm{DIF}| \sum_{i=1}^{f} \sum_{j=1}^{n_{i}}\left(\omega_{i, j}\left|L_{\pi(i, j)}\right|+\right.$ $\left.\omega_{i, 0} d_{\pi(i, j)}\right)$.

Theorem 1. The problems $1|\mathrm{GT}, \mathrm{CON}| \sum_{i=1}^{f}\left(\sum_{j=1}^{n_{i}} \omega_{i j}\left|L_{\pi(i, j)}\right|\right.$ $\left.+\omega_{i, 0} d_{i}^{\mathrm{opt}}\right), \quad 1|\mathrm{GT}, \mathrm{SLK}| \sum_{i=1}^{f}\left(\sum_{j=1}^{n_{i}} \omega_{i, j}\left|L_{\pi(i, j)}\right|+\omega_{i, 0} q_{i}^{\mathrm{opt}}\right)$, and $1|\mathrm{GT}, \mathrm{DIF}| \sum_{i=1}^{f} \sum_{j=1}^{n_{i}}\left(\omega_{i, j}\left|L_{\pi(i, j)}\right|+\omega_{i, 0} d_{\pi(i, j)}\right)$ can be solved in $O(n \log n)$ time, respectively.

Proof. Step 1 and Step 4 need time $O(n)$, respectively. Step 2 needs $O\left(\sum_{i=1}^{f} n_{i} \log n_{i}\right) \leq O(n \log n)$ time. Step 3 needs
$O(f \log f) \leq O(n \log n)$ time $\left(\sum_{i=1}^{f} n_{i}=n\right.$ and $\left.f<n\right)$. Thus, the total time complexity of Algorithm 1 is $O(n \log n)$.

Example 1. We only consider the problem 1|GT, CON $\mid \sum_{i=1}^{f}$ $\left(\sum_{j=1}^{n_{i}} \omega_{i j}\left|L_{\pi(i, j)}\right|+\omega_{i, 0} d_{i}^{\text {opt }}\right)$. Consider $n=12, f=3, G_{1}:\left[J_{1,1}\right.$, $\left.J_{1,2}, J_{1,3}\right], \quad p_{1,1}=2, p_{1,2}=4, p_{1,3}=6, \quad \omega_{1,1}=2, \quad \omega_{1,2}=4, \omega_{1,3}=$ $3, \omega_{1,0}=2, s_{1}=2 ; \quad G_{2}:\left[J_{2,1}, J_{2,2}, J_{2,3}, J_{2,4}\right], \quad p_{2,1}=5, p_{2,2}=8$, $p_{2,3}=4, p_{2,4}=3, \quad \omega_{2,1}=3, \omega_{2,2}=2, \quad \omega_{2,3}=2, \quad \omega_{2,4}=3, \omega_{2,0}=$ $1, s_{2}=4 ; \quad$ and $G_{3}:\left[J, J_{3,2}, J_{3,3}, J_{3,4}, J_{3,5}\right], \quad p_{3,1}=9, p_{3,2}=4$, $p_{3,3}=2, p_{3,4}=3, p_{3,5}=7, \quad \omega_{3,1}=4, \omega_{3,2}=2, \quad \omega_{3,3}=5, \omega_{3,4}=3$, $\omega_{3,5}=7, \omega_{3,0}=5, s_{3}=3$.

The solution is as follows:

Step 1: we calculate $k_{1}=2, W_{1,1}=2, W_{1,2}=4, W_{1,3}=3$; $k_{2}=2, W_{2,1}=1, W_{2,2}=4, W_{2,3}=5, W_{2,4}=3$; and $k_{3}=$ $3, W_{3,1}=5, W_{3,2}=9, W_{3,3}=11, W_{3,4}=10, W_{3,5}=7$

Step 2: sequence of jobs within each group: $G_{1}$ : $\left[J_{1,3} \longrightarrow J_{1,1} \longrightarrow J_{1,2}\right], \quad G_{2}:\left[J_{2,2} \longrightarrow J_{2,3} \longrightarrow J_{2,4}\right.$ $\left.\longrightarrow J_{2,1}\right]$, and $G_{3}:\left[J_{3,1} \longrightarrow J_{3,2} \longrightarrow J_{3,3} \longrightarrow J_{3,4}\right.$ $\left.\longrightarrow J_{3,5}\right]$ 
Step 1: for the problem $1|\mathrm{GT}, \mathrm{CON}| \sum_{i=1}^{f}\left(\sum_{j=1}^{n_{i}} \omega_{i j}\left|L_{\pi(i, j)}\right|+\omega_{i, 0} d_{i}^{\text {opt }}\right)$, we calculate $k_{i}$ of each group according to Lemma 3 and calculate $W_{i, j}$ according to equation (19). For the problem $1|\mathrm{GT}, \mathrm{SLK}| \sum_{i=1}^{f}\left(\sum_{j=1}^{n_{i}} \omega_{i, j}\left|L_{\pi(i, j)}\right|+\omega_{i, 0} q_{i}^{\text {opt }}\right)$, we calculate $l_{i}$ of each group according to Lemma 4 and calculate $V_{i, j}$ according to equation (21). For the problem $1|\mathrm{GT}, \mathrm{DIF}| \sum_{i=1}^{f} \sum_{j=1}^{n_{i}}\left(\omega_{i, j}\left|L_{\pi(i, j))}\right|+\omega_{i, 0} d_{\pi(i, j)}\right)$, we calculate $U_{i, j}$ according to equation (23).

Step 2: we assign the smallest $W_{i, j}\left(V_{i, j}\right.$ and $\left.U_{i, j}\right)$ value to the job with the largest $p_{i, j}$ value, the second smallest $W_{i, j}\left(V_{i, j}\right.$ and $\left.U_{i, j}\right)$ value to the job with the second largest $p_{i, j}$ value, and so on, with ties broken arbitrarily, and then obtain the internal job sequence of each group.

Step 3: we arrange the groups in nondecreasing order of $\theta_{i}$ by Lemma 7.

Step 4: according to Lemma 3, we calculate $d_{i}^{\text {opt }}$ for the problem $1|\mathrm{GT}, \mathrm{CON}| \sum_{i=1}^{f}\left(\sum_{j=1}^{n_{i}} \omega_{i j}\left|L_{\pi(i, j)}\right|+\omega_{i, 0} d_{i}^{\text {opt }}\right)$. According to Lemma 4, we calculate $q_{i}^{\text {opt }}$ for the problem $1|\mathrm{GT}, \operatorname{SLK}| \sum_{i=1}^{f}\left(\sum_{j=1}^{n_{i}} \omega_{i, j}\left|L_{\pi(i, j)}\right|+\omega_{i, 0} q_{i}^{\text {opt }}\right)$. According to Lemma 6 , we calculate $d_{i, j}$ for the problem $1|\mathrm{GT}, \mathrm{DIF}| \sum_{i=1}^{f} \sum_{j=1}^{n_{i}}\left(\omega_{i, j}\left|L_{\pi(i, j)}\right|+\omega_{i, 0} d_{\pi(i, j)}\right)$.

Algorithm 1: Optimal algorithm.

Step 3: $\theta_{1}=7, \theta_{2}=24, \theta_{3}=28 / 5$, and $\theta_{3}<\theta_{1}<\theta_{2}$; hence, the optimal group sequence is $\left[G_{3}, G_{1}, G_{2}\right]$

Step 4: $d_{3}^{\text {opt }}=s_{3}+\sum_{j=1}^{k_{3}} p_{3 j}=18, d_{1}^{\text {opt }}=S_{1}+s_{1}+\sum_{j=1}^{k_{1}}$ $p_{1 j}=28+2+8=38$, and $d_{2}^{\mathrm{opt}}=S_{2}+s_{2}+\sum_{j=1}^{k_{2}} p_{2 j}=$ $42+4+12=58$

\section{Conclusions}

In this paper, we studied the single-machine scheduling problem involving the due-date assignment and job scheduling under the group technology. The due dates are assignable according to one of the following three due-date assignment methods: CON, SLK, and DIF due-date assignment. The objective is to find the optimal due dates of jobs, a sequence for groups, and jobs to minimize a total cost function. We show that the problem can be solved in polynomial time. In future study, we can consider the group scheduling models associated with learning and deteriorating effects. In addition, we can further study the group scheduling with CON and SLK due-date assignment in the flow shop setting.

\section{Data Availability}

No data were used to support this study.

\section{Conflicts of Interest}

The authors declare that they have no conflicts of interest.

\section{Acknowledgments}

This paper was supported by the National Natural Science Foundation of China (grant nos. 71871091 and 71471057) and the Natural Science Foundation of Liaoning Province in China (grant no. 2020-MS-233).

\section{References}

[1] J. S. Neufeld, J. N. D. Gupta, and U. Buscher, "A comprehensive review of flowshop group scheduling literature," Computers \& Operations Research, vol. 70, pp. 56-74, 2016.
[2] L. Yang, Y. Zhao, and X. Ma, "Group maintenance scheduling for two-component systems with failure interaction," Applied Mathematical Modelling, vol. 71, pp. 118-137, 2019.

[3] S.-J. Yang and D.-L. Yang, "Single-machine scheduling simultaneous with position-based and sum-of-processingtimes-based learning considerations under group technology assumption," Applied Mathematical Modelling, vol. 35, no. 5, pp. 2068-2074, 2011.

[4] Y.-Y. Lu, J.-J. Wang, and J.-B. Wang, "Single machine group scheduling with decreasing time-dependent processing times subject to release dates," Applied Mathematics and Computation, vol. 234, pp. 286-292, 2014.

[5] N. Yin, L. Kang, and X.-Y. Wang, "Single-machine group scheduling with processing times dependent on position, starting time and allotted resource," Applied Mathematical Modelling, vol. 38, no. 19-20, pp. 4602-4613, 2014.

[6] J.-J. Wang and Y.-J. Liu, "Single-machine bicriterion group scheduling with deteriorating setup times and job processing times," Applied Mathematics and Computation, vol. 242, pp. 309-314, 2014.

[7] J.-B. Wang and J.-J. Wang, "Single machine group scheduling with time dependent processing times and ready times," Information Sciences, vol. 275, pp. 226-231, 2014.

[8] H. Qin, Z.-H. Zhang, and D. Bai, "Permutation flowshop group scheduling with position-based learning effect," Computers \& Industrial Engineering, vol. 92, pp. 1-15, 2016.

[9] Y.-Y. Lu, J.-B. Wang, P. Ji, and H. He, "A note on resource allocation scheduling with group technology and learning effects on a single machine," Engineering Optimization, vol. 49, no. 9, pp. 1621-1632, 2017.

[10] X. Zhang, L. Liao, W. Zhang, T. C. E. Cheng, Y. Tan, and M. Ji, "Single-machine group scheduling with new models of position-dependent processing times," Computers \& Industrial Engineering, vol. 117, pp. 1-5, 2018.

[11] F. Liu, J. Yang, and Y.-Y. Lu, "Solution algorithms for singlemachine group scheduling with ready times and deteriorating jobs," Engineering Optimization, vol. 51, no. 5, pp. 862-874, 2019.

[12] J.-B. Wang and X.-X. Liang, "Group scheduling with deteriorating jobs and allotted resource under limited resource availability constraint," Engineering Optimization, vol. 51, no. 2, pp. 231-246, 2019.

[13] Y. Yin, T. C. E. Cheng, C.-C. Wu, and S.-R. Cheng, "Singlemachine common due-date scheduling with batch delivery costs and resource-dependent processing times," International Journal of Production Research, vol. 51, no. 17, pp. 5083-5099, 2013. 
[14] Y. Yin, M. Liu, T. C. E. Cheng, C.-C. Wu, and S.-R. Cheng, "Four single-machine scheduling problems involving due date determination decisions," Information Sciences, vol. 251, pp. 164-181, 2013.

[15] D.-J. Wang, Y. Yin, J. Xu, W.-H. Wu, S.-R. Cheng, and C.-C. $\mathrm{Wu}$, "Some due date determination scheduling problems with two agents on a single machine," International Journal of Production Economics, vol. 168, pp. 81-90, 2015.

[16] D. Shabtay, "Optimal restricted due date assignment in scheduling," European Journal of Operational Research, vol. 252, no. 1, pp. 79-89, 2016.

[17] S. Li, C. T. Ng, and J. Yuan, "Group scheduling and due date assignment on a single machine," International Journal of Production Economics, vol. 130, no. 2, pp. 230-235, 2011.

[18] W. Liu, X. Hu, and X. Wang, "Single machine scheduling with slack due dates assignment," Engineering Optimization, vol. 49, no. 4, pp. 709-717, 2017.

[19] J.-B. Wang, X.-N. Geng, L. Liu, J.-J. Wang, and Y.-Y. Lu, "Single machine CON/SLK due date assignment scheduling with controllable processing time and job-dependent learning effects," The Computer Journal, vol. 61, no. 9, pp. 1329-1337, 2018.

[20] M. Ji, K. Chen, J. Ge, and T. C. E. Cheng, "Group scheduling and job-dependent due window assignment based on a common flow allowance," Computers \& Industrial Engineering, vol. 68, pp. 35-41, 2014.

[21] P. Brucker, Scheduling Algorithms, Springer, Berlin, Germany, 3rd edition, 2001.

[22] L. Sun, A. J. Yu, and B. Wu, "Single machine common flow allowance group scheduling with learning effect and resource allocation," Computers and Industrial Engineering, vol. 139, Article ID 106126, 2020.

[23] R. L. Graham, E. L. Lawler, J. K. Lenstra, and A. H. G. R. Kan, "Optimization and approximation in deterministic sequencing and scheduling: a survey," Annals of Discrete Mathematics, vol. 5, pp. 287-326, 1979.

[24] G. H. Hardy, J. E. Littlewood, and G. Polya, Inequalities, Cambridge University Press, Cambridge, UK, 2nd edition, 1967. 